\title{
Quantifying the impact of vaccine hesitancy in prolonging the need for Non-Pharmaceutical Interventions to control the COVID-19 pandemic
}

Daniela Olivera Mesa ( $\nabla$ d.olivera-mesa17@imperial.ac.uk)

Imperial College London https://orcid.org/0000-0002-6190-2855

Alexandra Hogan

Imperial College London https://orcid.org/0000-0002-6271-9921

Oliver Watson

Imperial College London https://orcid.org/0000-0003-2374-0741

Giovanni Charles

Imperial College London

Katharina Hauck

Imperial College London

Azra C Ghani

Imperial College London

Peter Winskill

Imperial College London

\section{Letter}

Keywords: immunology, COVID-19, SARS-CoV-2, vaccine hesitancy

Posted Date: March 26th, 2021

DOI: https://doi.org/10.21203/rs.3.rs-343127/v1

License: (c) (i) This work is licensed under a Creative Commons Attribution 4.0 International License. Read Full License

Version of Record: A version of this preprint was published at Communications Medicine on February 10th, 2022. See the published version at https://doi.org/10.1038/s43856-022-00075-x. 


\section{Abstract}

Vaccine hesitancy - a delay in acceptance or refusal of vaccines despite availability - has the potential to threaten the successful roll-out of SARS-CoV-2 vaccines globally. Here, we evaluate the potential impact of vaccine hesitancy on the control of the pandemic and the relaxation of non-pharmaceutical interventions (NPIs) by combining an epidemiological model of SARS-CoV-2 transmission with data on vaccine hesitancy from population surveys. Our findings suggest that the mortality over a 2-year period could be up to 8 times higher in countries with high vaccine hesitancy compared to an ideal vaccination uptake if NPIs are relaxed. Alternatively, high vaccine hesitancy could prolong the need for NPIs to remain in place. Addressing vaccine hesitancy with behavioural interventions is therefore an important priority in the control of the COVID-19 pandemic.

\section{Main Text}

The COVID-19 pandemic has simultaneously resulted in high global mortality and major economic disruptions. As a control measure, non-pharmaceutical interventions (NPIs) such as social distancing and mobility restrictions have been put in place worldwide and have successfully reduced transmission of the virus. However, these interventions are unsustainable in the long-term ${ }^{4}$ and current hopes to control the pandemic rely heavily on vaccination.

In December 2020, the first vaccine against SARS-CoV-2 was approved; by March 2021, 12 vaccines had been licensed (https://vac-lshtm.shinyapps.io/ncov_vaccine_landscape/) and more than 300 million vaccination doses administered worldwide (https://ourworldindata.org/covid-vaccinations\#). Their reported efficacy against symptomatic disease ranges from $50 \%$ to over $95 \%{ }^{5-9}$. Given the high basic reproduction number for SARS-CoV-2 (estimates range between 3-4) ${ }^{4}$ high levels of vaccine uptake will be required to achieve herd immunity ${ }^{3}$, particularly if children are not vaccinated during the first phase of rollout.

One major concern that threatens to limit the impact of vaccination is vaccine hesitancy ${ }^{2}$. Population surveys have found that between $14 \%^{10}$ and $27 \%^{11}$ of adults say that they will not accept a vaccine if available, whilst between $14 \%{ }^{10}$ and $19 \%^{11}$ say that they are uncertain. There is a large variation in vaccine hesitancy between countries, with the proportion saying that they would get a SARS-Cov-2 vaccine if it became available, ranging from $40 \%$ for France ${ }^{11}$ to $89 \%$ for China ${ }^{10}$. In many countries, vaccine hesitancy is heterogenous across sub-populations depending on gender, age, ethnicity, religion, or socioeconomic status ${ }^{10-12}$. The key drivers of SARS-CoV-2 vaccine hesitancy are related to concerns about the accelerated pace of vaccine development ${ }^{12}$, side-effects ${ }^{11}$, and the spread of misinformation about the pandemic ${ }^{2}$.

To understand the likely impact of vaccine hesitancy on future control of the pandemic, we use a mathematical model of SARS-CoV-2 transmission ${ }^{3}$ to explore vaccine hesitancy through its impact on 
population coverage. We capture the effect of reduced coverage using three measured levels of vaccine hesitancy based on self-reported intention to be vaccinated from recent behavioural survey data ${ }^{11}$. Low, medium, and high hesitancy levels result in optimistic, neutral and pessimistic scenarios respectively. Hesitancy levels are further disaggregated by age-group to capture variation in attitudes with respect to age. The hesitancy scenarios are compared to an ideal counterfactual assuming no vaccine hesitancy, in which we assume that a small proportion ( $2 \%$ ) of the population will be ineligible to be vaccinated due to contraindications. We model each scenario with both a high and a moderate vaccine efficacy profile that represents the range of efficacies of currently approved vaccines. Informed by current vaccine roll-out in high-income countries, we assume that vaccination started in January 2021 and is implemented at a rate that results in a total campaign of 10 months to fully vaccinate the population above 15 years old, with no vaccine hesitancy (the duration of the campaign is shorter in scenarios with vaccine hesitancy, due to assuming the same roll-out speed but lower uptake).

We first sought to determine the public health impact of vaccination and vaccine hesitancy as NPIs are lifted. To do so, we allowed the time-varying reproductive number in the absence of immunity $R_{t}$, to be increased in steps such that the effective reproductive number $R_{\text {eff }}$, accounting for vaccine-induced immunity, remains at the threshold value of 1 with the assumption of ideal vaccination uptake (Figure 1 a, c). In this ideal scenario, NPIs can be fully lifted at the end of the vaccination period with a high efficacy vaccine ( $94 \%$ efficacy, Figure 1 a). However, with a moderate efficacy vaccine (63\% efficacy), some NPIs or other population-level behavioural changes may need to remain to control the epidemic (Figure $1 \mathrm{c}$ ). In the presence of vaccine hesitancy, lifting NPIs and relying on vaccine-induced immunity for control is predicted to lead to periodic outbreaks determined by the duration of naturally induced immunity, with the outbreak size greater at higher levels of vaccine hesitancy (Figure $1 \mathrm{~b}, \mathrm{~d}$ ). For a high efficacy vaccine, even under the optimistic vaccine hesitancy scenario, daily deaths per million at the peak of the first outbreak are projected to be 8.7 times higher than under the ideal scenario (Figure 1b). This translates to a cumulative impact of 236 more deaths per million population in the two years after vaccination begins. For a vaccine of moderate efficacy, in an optimistic vaccine hesitancy scenario, the cumulative impact of vaccine hesitancy is projected to lead to 305 extra deaths per million population over this period. These adverse impacts of vaccine hesitancy on transmission, symptomatic disease, hospitalisations, and deaths affect vaccinated as well as unvaccinated individuals because of imperfect vaccine efficacy (Figure 2). Under the pessimistic scenario for vaccine hesitancy, the resulting lower vaccination coverage is projected to lead to a $33 \%$ and $46 \%$ increase in hospitalisations in the vaccinated population for the high and moderate vaccine efficacy profile, respectively, and a $18 \%$ and $46 \%$ increase in deaths in the vaccinated population, compared to an ideal vaccination scenario (Figure 2).

As an alternative way to assess the impact of vaccine hesitancy on the pandemic, we evaluated the degree to which other NPIs would need to remain in place given the real-time achieved vaccine coverage in order to prevent further epidemics (i.e. keep $R_{\text {eff }}<1$, Figure 3 ). For the high efficacy vaccine, under the ideal scenario, we predict that NPIs could be fully lifted by the end of 2021 whilst keeping transmission under control. However, even under the optimistic scenario with low vaccine hesitancy, limited NPIs or 
other behavioural modifications might need to remain in place, with Rt having to remain below 2.4 to prevent further epidemics, $79 \%$ of the assumed R0 of 3 . For the more pessimistic scenarios of vaccine hesitancy, and for lower vaccine efficacy, these differences become more pronounced. A difference of $\sim 35 \%$ in the effective reproductive number could represent the closure of educational institutions or limiting interaction between households to achieve control of the epidemic ${ }^{13}$; both of which are not sustainable or desirable.

Our illustrative examples above are comparable to the waves of COVID-19 outbreaks in Europe. However, vaccine hesitancy varies between countries (Figure 4a). To evaluate the impact of these variations, we chose three European countries with different vaccine acceptance views: France, Germany, and the United Kingdom (UK). For each country, we modelled the trajectory of the pandemic under an ideal vaccination and a neutral vaccine hesitancy scenario. For a vaccine with high efficacy, we project 1.3, 4.5 and 8.7 times more deaths in 2021/2022 in a scenario with hesitancy compared to an ideal scenario in the UK, Germany and France respectively (Figure 4).

ur findings show the considerable impact of vaccine hesitancy, detailing the considerable mortality that could be averted with increased vaccine coverage. However, our analysis necessarily makes many simplifying assumptions, and it is important to note that the future trajectory of the epidemic will depend on the complex interactions between vaccination uptake, behaviour, and government interventions. First, we have assumed homogenous mixing between vaccine hesitant individuals. However, as has been seen for other diseases, COVID-19 vaccine hesitancy is heterogenous and clustered within population subgroups ${ }^{14}$. Transmission is more likely to be sustained within clusters with low vaccine coverage ${ }^{15,16}$ and therefore future outbreaks may be limited to these sub-populations. Secondly, self-reported attitudes to COVID-19 vaccines are changing over time ${ }^{10,11}$ and levels may improve as confidence grows in the vaccination programmes. Thirdly, we do not account for immune escape from the vaccine due to new variants arising. Whilst second generation vaccines will likely become available to address this issue, it is currently unclear whether some of the high levels of vaccine uptake observed in early vaccine roll-outs would be sustained in subsequent booster programmes.

Getting vaccinated is an individual choice, but these individual choices have population wide effects that are likely to challenge current efforts to control COVID-19. Our findings suggest that vaccine hesitancy may have a substantial impact on the pandemic trajectory, deaths, and hospitalization. To prevent such adverse outcomes, NPIs would need to stay in place longer, or possibly indefinitely, resulting in high economic and social costs 17,18 . Reducing vaccine hesitancy is therefore an important public health priority. Interventions that aim to build trust, for example with community-based public education or via positive role-models, are proven efficacious approaches to address hesitancy ${ }^{19}$. There is an ongoing debate about vaccine passports as a condition to travel, or a vaccination requirement for employees ${ }^{20}$. Such interventions may be effective because they incentivize individuals to get vaccinated, but they are controversial in libertarian democracies because they curtail personal freedom and individual choice about medical treatments. The alternative will be to accept some level of disease, hospitalisation and 
deaths given the level of vaccine coverage achieved whilst allowing NPIs to be lifted, given that NPIs are not a sustainable long-term method for control.

\section{Online Methods}

\section{Vaccine hesitancy data}

Attitudes towards COVID-19 vaccination were obtained from the Imperial College London YouGov Covid 19 Behaviour Tracker Data ${ }^{11}$. This data set includes weekly surveys about people's behaviours in response to COVID-19 (including vaccines) as well as standard demographic questions on age, gender, and household structure.

We extracted the survey results from February $8^{\text {th }}$ - February $15^{\text {th }}, 2021$ for 10 European countries. To assess vaccine hesitancy, we used data from one question pertaining to COVID-19 vaccine acceptance in which participants were asked to what extent they would definitely get a COVID-19 vaccine, if it became available to them next week. Answers were obtained on a numeric scale ranging from "Strongly agree -1 " to "Strongly disagree -5 ". We grouped survey results into 5-year age groups and categorised three vaccine acceptance levels for each group - high, middle, and low vaccine hesitancy - where vaccine acceptance was the proportion of individuals whose answers were " 1 ", "1 or 2 " and " 1,2 or 3 " respectively.

\section{Mathematical model}

We used a previously developed mathematical model for SARS-CoV-2 transmission and vaccination ${ }^{3}$. The age-structured deterministic SEIR-type compartmental model incorporates an age specific probability of infection determined by age-based contact matrices as well as the disease clinical pathway, which includes hospitalisation, oxygen support and intensive care needs. Vaccination is modelled as an additional dimension disaggregating the population into those who have not received the vaccine, have received the vaccine but are not yet protected, have received the vaccine and are protected and those who have received the vaccine but are no-longer protected (if vaccine-derived immunity is not life-long). In this model only those who are currently infected do not receive the vaccine. Protection due to vaccination is modelled at two stages in the model; 1 ) reducing the probability of infection upon exposure (efficacy against infection) and 2) reducing the probability of hospitalisation being indicated after developing disease (efficacy against hospitalisation and death).

Parameters for SARS-CoV-2 infection, health care capacity, age-distribution and contact patterns are based on previous work ${ }^{3,21}$. Vaccine induced immunity was assumed lifelong, while natural immunity was assumed to last for an average of one year ${ }^{22}$. To produce simulations representing the different vaccines approved to date, each scenario was run for two vaccines: one with high efficacy ( $94 \%$ efficacy against infection $\left.{ }^{5}\right)$ ) and one with moderate efficacy ( $63 \%$ efficacy against infection ${ }^{6}$ ). For both vaccines we assume an additional $60 \%$ efficacy against hospitalisation for breakthrough infections, resulting in an overall vaccine efficacy against hospitalisation and death of $98 \%$ for the high efficacy vaccine and $85 \%$ 
for the moderate efficacy vaccine. A summary of key parameters is given in Table S1. The model code is freely available at https://github.com/mrc-ide/nimue.

To mimic current vaccine rollout plans, vaccination is introduced in the population at the beginning of January 2021. We assumed that only individuals aged 15 years and above are vaccinated and that vaccination occurs at a constant rate that results in $98 \%$ of the adult population and hence $80 \%$ of the population being vaccinated after ten months under the ideal vaccine hesitancy scenario. For other vaccine hesitancy scenarios, we implement the same rate of vaccination (given that this is constrained not by vaccine uptake but by the supply and delivery of vaccines) such that the final lower level of coverage is achieved earlier than ten months. Vaccines are allocated prioritising older age groups first: with $80+$ years vaccinated first and then sequentially including additional age groups in 5-year age-bands down to 15-19 years.

\section{Simulations}

To simulate a representative pre-vaccination scenario, we generated a scenario in which Rt was 2.5 up to April 2020, subsequently decreased to 1 to represent the impact of NPIs against the first wave, and then rose to 1.5 during the latter half of 2020 to represent a second wave. Following the introduction of vaccination in January 2021, we set Rt to increase in 5 fixed steps. The time for each step increase was determined by estimating when vaccination coverage had reached levels such that the effective reproduction number in the presence of vaccination was one $\left(R_{\text {eff }}=1\right)$. This coverage was estimated based on the following equation:

Coverage $*$ efficacy $=1-\frac{1}{R_{t}}$

At the end of the vaccination period, $R_{t}$ remained at a value such that the effective reproduction number continued at the threshold value of 1 , taking into account final vaccination coverage and vaccine efficacy.

We consider four potential scenarios for vaccine coverage per age group:

1. Ideal: $98 \%$ coverage for all age groups $15+$.

2. Optimistic: Final coverage per age group is given by vaccine acceptance from the low vaccine hesitancy level.

3. Neutral: Final coverage per age group is given by vaccine acceptance from the middle vaccine hesitancy level.

4. Pessimistic: Final coverage per age group is given by vaccine acceptance from the high vaccine hesitancy level. 
When analysing the impact of lifting NPIs, the Rt profile following the introduction of vaccination was generated based on an ideal scenario for vaccination uptake. Conversely, when evaluating the degree to which NPIs would need to remain in place, the Rt profile after the introduction of vaccination was set up based on each one of the vaccine coverage scenarios described above.

Simulations for the representative scenario were run using a U.K representative contact matrix (https://github.com/sbfnk/socialmixr) and epidemics were seeded at the end February 2020 with 20 cases. As an output for each simulation, we estimated the number of deaths and hospitalisations associated with COVID-19 over the two-year period from 1 January 2021 to 31 December 2022.

To generate country specific simulations, we parameterise the model with data on the population size and age distribution of the country (https://population.un.org/wpp/) and representative contact matrices obtained from a systematic review of social contact surveys through the socialmixR package (https://github.com/sbfnk/socialmixr) . The model was then fitted to reported daily cases and deaths up to December $31^{\text {st }}, 2020$ by varying three parameters - the start date of the epidemic, the initial R0 and the effect size of changes in mobility on transmission (using mobility data from Google (https://www.google.com/covid19/mobility )). Model fitting was performed using a Metropolis Hastings MCMC based sampling scheme as previously described ${ }^{23}$. The resulting fit generates an Rt trajectory up to the introduction vaccination in January 2021, after which, Rt was set to increase by 10 fixed steps, up to the theoretical herd immunity threshold based on an ideal vaccination schedule (as described above). The pandemic trajectory was evaluated for two scenarios: An ideal scenario and a neutral vaccine hesitancy scenario (as described above), using country specific data on vaccine hesitancy and demography.

\section{Declarations}

\section{Data availability}

All data used in this study are from publicly accessible sources and the results generated are available from the corresponding author on reasonable request.

\section{Acknowledgements}

All authors acknowledge funding from the MRC Centre for Global Infectious Disease Analysis (reference MR/R015600/1), jointly funded by the UK Medical Research Council (MRC) and the UK Foreign, Commonwealth \& Development Office (FCDO), under the MRC/FCDO Concordat agreement and is also part of the EDCTP2 programme supported by the European Union. $\mathrm{KH}$ acknowledges funding by Community Jameel. PW and $\mathrm{ABH}$ acknowledge funding from the Imperial College Research Fellowship scheme. ACG, DOM and GDC acknowledge funding from The Wellcome Trust (no. 215143/Z/18/Z to DOM).

\section{Author contributions}


ACG, KH, PW and DOM conceived the study. ABH, PW, OJW and GDC developed and coded the model. DOM ran the simulations and undertook the analysis with support from PW. OJW parametrised the model to country data. DOM produced the first draft of the manuscript with additional input from $\mathrm{PW}, \mathrm{KH}$ and ACG. All authors approved the final version for submission.

\section{Competing interests}

$A B H, P W$ and ACG declare consultancy fees from the World Health Organization in relation to modelling COVID-19 vaccine impact in the European region, outside the submitted work. The authors declare no other competing interests.

\section{References}

1. MacDonald, N.E. Vaccine hesitancy: Definition, scope and determinants. Vaccine 33, 4161-4164 (2015).

2. Loomba, S., de Figueiredo, A., Piatek, S.J., de Graaf, K. \& Larson, H.J. Measuring the impact of COVID19 vaccine misinformation on vaccination intent in the UK and USA. Nature Human Behaviour (2021).

3. Hogan, A.B., et al. Report 33: Modelling the allocation and impact of a COVID-19 vaccine. in COVID19 reports (Imperial College London, 2020).

4. Flaxman, S., et al. Estimating the effects of non-pharmaceutical interventions on COVID-19 in Europe. Nature 584, 257-261 (2020).

5. Polack, F.P., et al. Safety and Efficacy of the BNT162b2 mRNA Covid-19 Vaccine. New England Journal of Medicine 383, 2603-2615 (2020).

6. Voysey, M., et al. Safety and efficacy of the ChAdOx1 nCoV-19 vaccine (AZD1222) against SARSCoV-2: an interim analysis of four randomised controlled trials in Brazil, South Africa, and the UK. The Lancet 397, 99-111 (2021).

7. Baden, L.R., et al. Efficacy and Safety of the mRNA-1273 SARS-CoV-2 Vaccine. New England Journal of Medicine 384, 403-416 (2020).

8. Logunov, D.Y., et al. Safety and efficacy of an rAd26 and rAd5 vector-based heterologous prime-boost COVID-19 vaccine: an interim analysis of a randomised controlled phase 3 trial in Russia. The Lancet.

9. Sinovac Biotech Ltd. Sinovac Announces Phase III Results of Its COVID-19 Vaccine. (2021).

10. Lazarus, J.V., et al. A global survey of potential acceptance of a COVID-19 vaccine. Nature Medicine 27, 225-228 (2021).

11. Jones, S.P., Imperial College London \& YouGov Plc. Imperial College London YouGov Covid Data Hub. (YouGov Plc, 2020).

12. Freeman, D., et al. COVID-19 vaccine hesitancy in the UK: the Oxford coronavirus explanations, attitudes, and narratives survey (Oceans) II. Psychological Medicine, 1-15 (2020). 
13. Brauner, J.M., et al. Inferring the effectiveness of government interventions against COVID-19. Science 371, eabd9338 (2021).

14. de Figueiredo, A. Sub-national forecasts of COVID-19 vaccine acceptance across the UK: a largescale cross-sectional spatial modelling study. in medRxiv 2020.2012.2017.20248382 (2020).

15. Truelove, S.A., et al. Characterizing the impact of spatial clustering of susceptibility for measles elimination. Vaccine 37, 732-741 (2019).

16. Salathé, M. \& Bonhoeffer, S. The effect of opinion clustering on disease outbreaks. Journal of the Royal Society, Interface 5, 1505-1508 (2008).

17. Nicola, M., et al. The socio-economic implications of the coronavirus pandemic (COVID-19): A review. International journal of surgery (London, England) 78, 185-193 (2020).

18. Mandel, A. \& Veetil, V. The Economic Cost of COVID Lockdowns: An Out-of-Equilibrium Analysis. Economics of Disasters and Climate Change 4, 431-451 (2020).

19. Vergara, R.J.D., Sarmiento, P.J.D. \& Lagman, J.D.N. Building public trust: a response to COVID-19 vaccine hesitancy predicament. Journal of Public Health (2021).

20. Brown, R.C.H., Kelly, D., Wilkinson, D. \& Savulescu, J. The scientific and ethical feasibility of immunity passports. The Lancet Infectious Diseases 21, e58-e63 (2021).

21. Walker, P.G.T., et al. The impact of COVID-19 and strategies for mitigation and suppression in lowand middle-income countries. Science 369, 413-422 (2020).

22. Hall, V., et al. Do antibody positive healthcare workers have lower SARS-CoV-2 infection rates than antibody negative healthcare workers? Large multi-centre prospective cohort study (the SIREN study), England: June to November 2020. medRxiv, 2021.2001.2013.21249642 (2021).

23. Watson, O.J., et al. Report 31: Estimating the burden of COVID-19 in Damascus, Syria: an analysis of novel data sources to infer mortality under-ascertainment in COVID-19 reports 1-46 (Imperial College London, 2020).

\section{Figures}



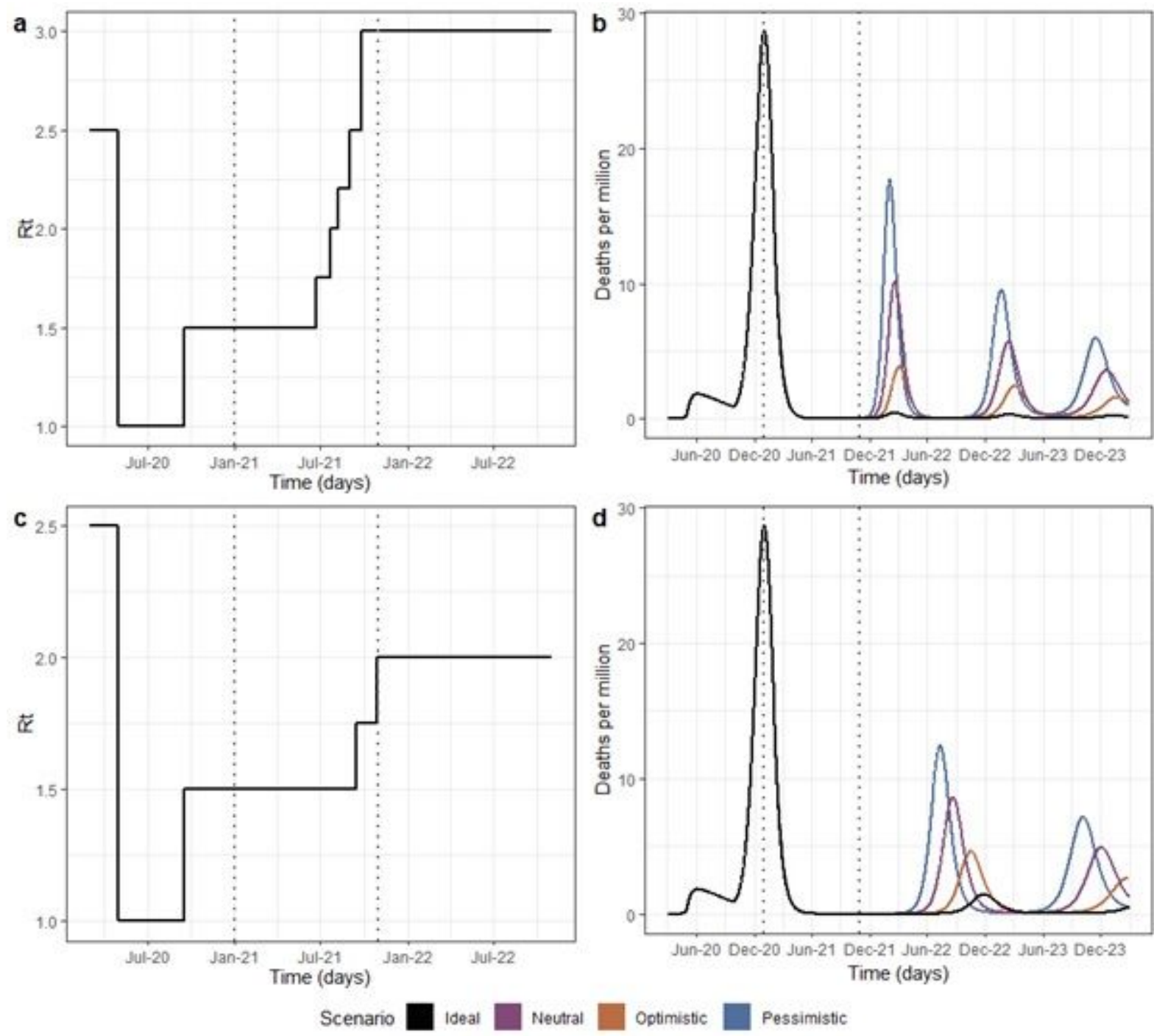

\section{Figure 1}

Projected COVID-19 dynamics for different vaccine hesitancy levels. Panels a-b show a high vaccine efficacy (94\% against infection, 98\% against hospitalisation and death), panels c-d moderate vaccine efficacy (63\% against infection, $85 \%$ against hospitalisation and death). Panels a and c show the reproductive number Rt profile, which represents the level of NPI stringency, with lower numbers indicating higher stringency. In this illustrative example, we assume that a first wave of transmission occurred at the beginning of 2020 with an Rt of 2.5. This was followed by NPIs leading to a reduction in Rt to 1 , followed by an Rt of 1.5 as NPIs are lifted leading to a second wave of transmission in the latter half of 2020. After vaccination is introduced at the beginning of 2021, NPIs in all scenarios are lifted according to a schedule based on coverage under the ideal scenario (no vaccine hesitancy, $98 \%$ of individuals 15 years plus are vaccinated). Panels $b$ and $d$ show projected deaths per million under four vaccine hesitancy scenarios: Ideal (no hesitancy, black), optimistic (low hesitancy, orange), neutral (medium hesitancy, purple), pessimistic (high hesitancy, blue). In each scenario, final vaccination coverage per age group and deaths vary according to vaccine hesitancy. Dashed lines indicate the vaccination rollout period in the ideal scenario. 

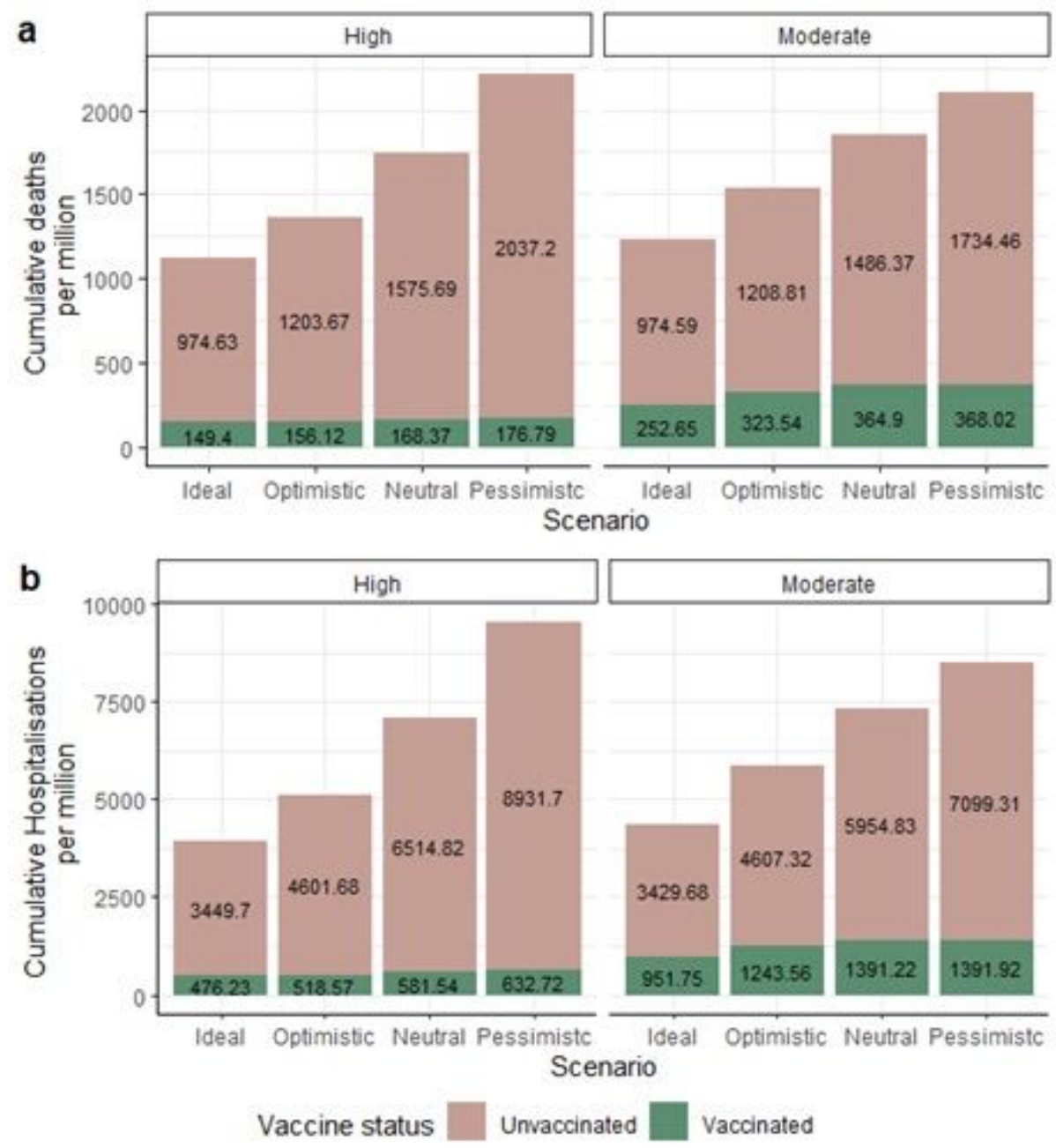

\section{Figure 2}

Public health impact of vaccine hesitancy. High vaccine efficacy is shown on the left and moderate vaccine efficacy on the right. The annotated numbers are the cumulative deaths a) and hospitalisations b) per million individuals for the vaccinated and unvaccinated populations at the end of the projection horizon (1 January 2021 - 31 December 2022). Vaccination coverage of individuals aged 15 years and older is highest in the ideal scenario at $98 \%$, and lowest in the pessimistic scenario because of vaccine hesitancy. 


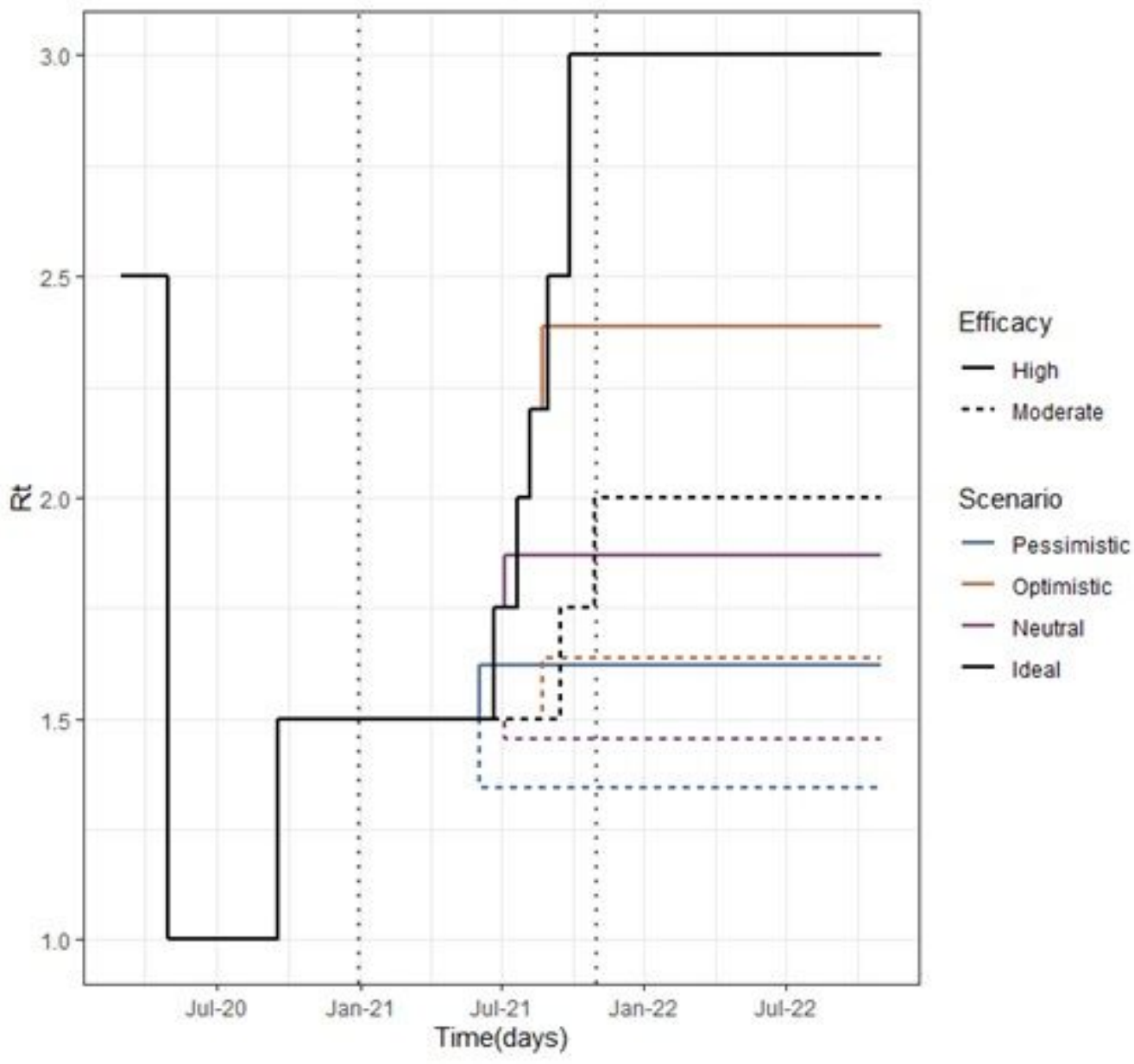

Figure 3

Stringency of NPIs required to control the epidemic under different vaccine hesitancy scenarios.

Reproductive number profiles are estimated to keep Reff $<1$ such that epidemic impact is the same for each scenario as in the ideal scenario. A lower reproductive number corresponds to more stringent NPIs. Continuous lines represent profiles for a high efficacy vaccine and dashed lines represent profiles for a moderate efficacy vaccine. The vertical dotted lines show the period of vaccination in the ideal scenario. 

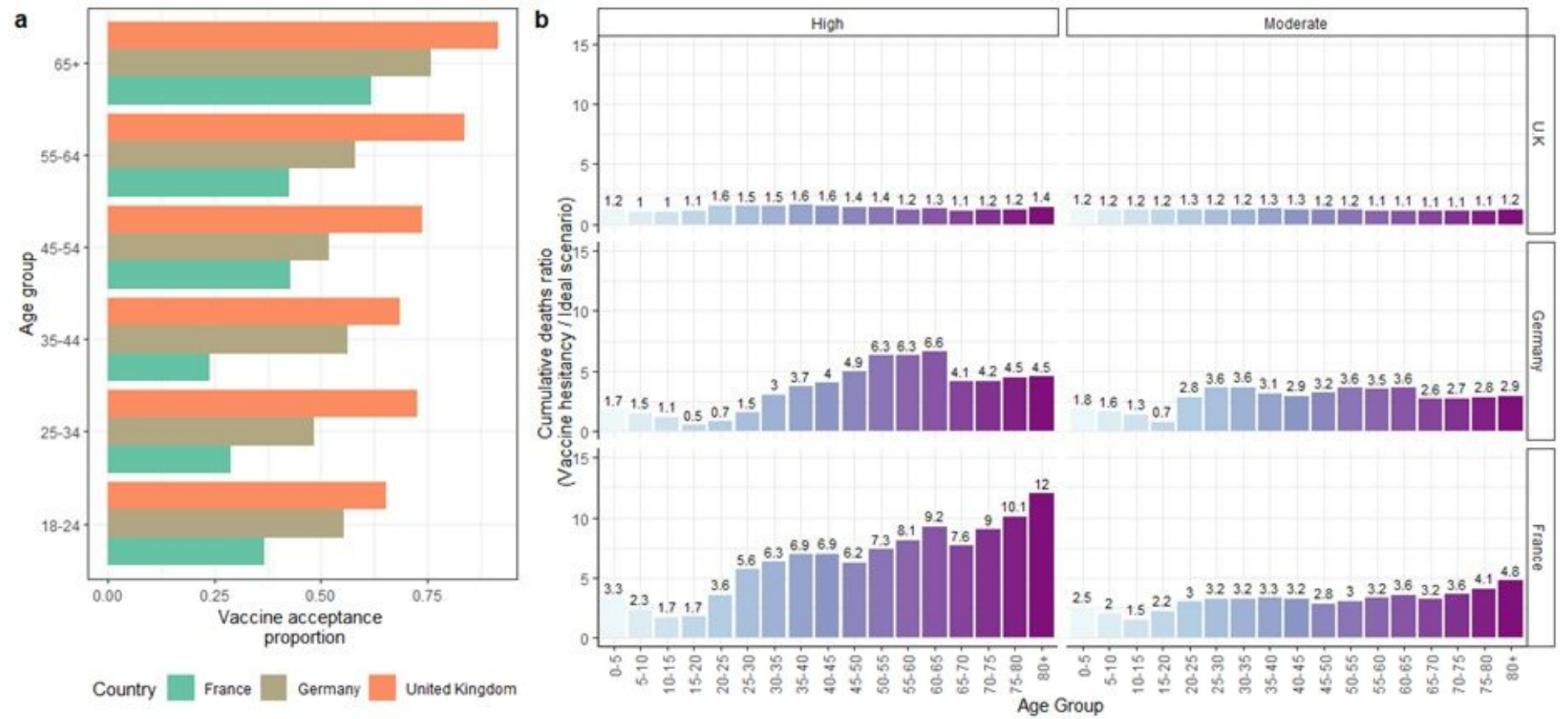

\section{Figure 4}

Impact of vaccine hesitancy for three European countries. a) Reported vaccine acceptance per age group in France, Germany and the United Kingdom reproduced from Jones et al.11. b) Cumulative death ratios per age group compared to the ideal vaccine uptake scenario, by country and vaccine efficacy profile. The ratio compares cumulative deaths projected over a two-year period after vaccination starts for two scenarios: An ideal scenario, where $98 \%$ of the population older than 15 years gets vaccinated and a vaccine hesitancy scenario, where coverage for people over 15 years old is based on vaccine acceptance from a). SARS-CoV-2 dynamics were fitted for each country for 2020 , simulations were generated for a vaccination campaign starting Jan 2021 and lasting 10 months.

\section{Supplementary Files}

This is a list of supplementary files associated with this preprint. Click to download.

- SupplementaryMaterial.docx 